\title{
A VARIATIONAL FORMULATION FOR A LEVEL SET REPRESENTATION OF MULTIPHASE FLOW AND AREA PRESERVING CURVATURE FLOW*
}

\author{
SELIM ESEDOḠLU ${ }^{\dagger}$ AND PETER SMEREKA
}

\begin{abstract}
Variational descriptions for various multiphase level set formulations involving curvature flow are discussed. A representation of $n$ phases using $n-1$ level set functions is introduced having the advantage that constraints preventing overlaps or vacuum are not needed. The representation is then used in conjunction with our variational formulation to deduce a novel level set based algorithm for multiphase flow. In addition, a similar variational formulation is applied to area preserving curvature flow. In this flow, the area (or volume in 3D) enclosed by each level set is preserved. Each algorithm has been implemented numerically and the results of such computations are shown.
\end{abstract}

Key words. level set, multiphase flow, variational

AMS subject classifications. $65 \mathrm{~K} 10$

\section{Introduction}

This paper is primarily concerned with the problem of of $n$ different materials, or phases, in which the $i$-th phase has a bulk energy density of $e_{i}$ (per unit area in two dimensions or per unit volume in three dimensions). In addition, the interface between phase $i$ and $j$, denoted $\Gamma_{i j}$ has a surface energy density (surface tension) of $f_{i j}$ (per unit length in two dimensions or area in three dimensions). We let $\boldsymbol{n}_{i j}$ denote the normal vector directed inward into phase $i$ from phase $j$. Then, for the model we consider, the normal speed of the interface to be given by

$$
v_{n}\left(\Gamma_{i j}\right)=f_{i j} \kappa_{i j}+e_{i}-e_{j}
$$

where $\kappa_{i j}$ is the curvature of $\Gamma_{i j}$; one has $\kappa_{i j}=-\nabla_{s} \cdot \boldsymbol{n}_{i j}$ where $\nabla_{s}$ is the surface gradient. We remark that for this formulation, if phase $i$ is say a circle of radius $R$ surrounded by phase $j$ then $\kappa_{i j}=1 / R$. The total energy of this system is given by

$$
E=\sum_{i<j}\left(\text { length of } \Gamma_{i j}\right) f_{i j}+\sum_{i}(\text { area of phase } i) e_{i} .
$$

As pointed out in $[15,23,24]$, gradient descent of (1.2) gives rise to (1.1). In addition, the condition that $\dot{E} \leq 0$ requires the following condition to be true at a triple point:

$$
\frac{\sin \theta_{1}}{f_{23}}=\frac{\sin \theta_{2}}{f_{13}}=\frac{\sin \theta_{3}}{f_{12}},
$$

where $\theta_{i}$ is the angle between the interfaces $\Gamma_{i j}$ and $\Gamma_{i k}$. In particular, if all the surface energy densities are equal, then the interfaces must intersect at $120^{\circ}$. See [23] for a derivation of this condition.

The above system has been previously studied. Reitich \& Soner examine the system in the limit of vanishing surface tension and argue that in this limit, the Jin.

*Received: July 9, 2007; accepted (in revised version): November 2, 2007. Communicated by Shi

$\dagger$ Department of Mathematics, University of Michigan, USA (esedoglu@umich.edu).

${ }_{\ddagger}^{\ddagger}$ Department of Mathematics, University of Michigan, USA (psmereka@umich.edu). 
system evolving by (1.1) gives rise to a unique solution. Their results are based on the concept of a weak viscosity solution. Merriman, Bence, \& Osher [10] extended the diffusion generated motion by mean curvature formulation to the case of multiple phases. In related work, Taylor [20] has provided a least time formulation in which existence and uniqueness of solutions has been established in the case in which the interfacial velocity is prescribed.

Zhao et al. [23, 24] introduced a level set description for this system. In their approach, each phase is represented as the zero level set of a function. Therefore, for $n$ different phases, $n$ different level set functions are needed. The problem with such a representation is that vacuum regions (which belong to none of the phases) or overlaps between different phases may occur. To remove this possibility the authors amend the variational principle with a constraint. There are several difficulties with their constraint based approach. The first is that when deriving the Euler-Lagrange equation, Dirac delta functions $\delta(\phi)$ of the level set function $\phi$ appear. The authors replace the $\delta(\phi)$ s using $|\nabla \phi|$ - a process they call rescaling. The second difficulty is that the no overlapping/no vacuum constraint influences the dynamics. In other words, the gradient descent of (1.2) with the no overlapping/no vacuum constraint will not necessarily give rise to (1.1). Another difficulty is that constraints incorporated into the variational formulation may introduce additional stiffness to the problem.

Another formulation was introduced by Smith et al. [19] in which they replaced the constraint with a projection step. However, it is not clear whether or not the projection step will affect the dynamics. In addition, there has been a variety of representations used in image processing applications which will be discussed in more detail in $\S 3$.

In this paper we remove the difficulties associated with constrained formulations. This is achieved by first reformulating the variational principle using a different inner product and including all the level sets in the energy functional. This variational formulation is known; see for example $[5,6,8]$. We, however, present it in a different way which helps motivate subsequent discussions. Next, we introduce a new representation for the $n$-phase problem using $(n-1)$ level set functions. The main advantage of this formulation is that no overlaps/no vacuum constraints are not needed.

We then combine our variational formulation and our new level set representation to deduce a formulation of the multiphase flow problem that is a gradient flow with respect to a particular inner product. The resulting level set equations are easily solved numerically using standard methods - a number of examples are presented. Another interesting level set evolution, in which each level curve moves by area preserving curvature flow, is also considered. We show that such a flow is a gradient flow with an infinite number of constraints. Then, as an application, we show that this flow can be used for the super-resolution of digital images.

\section{Mean curvature flow}

2.1. Lagrangian formulation. To begin, let us consider a closed curve, $\Gamma$, in the plane parameterized by $\alpha$. Thus we write

$$
\Gamma=\{\boldsymbol{x}(\alpha, t) \mid 0 \leq \alpha \leq 1\} .
$$

Let $\boldsymbol{n}$ be the inward-directed unit normal. The curvature of $\Gamma$ will be denoted $\kappa . \kappa$ and $\boldsymbol{n}$ are related by $\kappa=-\nabla_{s} \cdot \boldsymbol{n}$, where $\nabla_{s}$ is the surface divergence. Note that with this definition $\kappa=1 / R$ when $\Gamma$ is a circle of radius $R$. We say that $\Gamma$ is moving by 
curvature flow if

$$
\frac{d \boldsymbol{x}}{d t}=\kappa \boldsymbol{n} .
$$

Curves moving by curvature flow have a variational principle. To state this we first observe that the arc length of $\Gamma$ is

$$
L=\int_{0}^{1} \sqrt{x_{\alpha}^{2}+y_{\alpha}^{2}} d \alpha
$$

Then, we have the following well known theorem about curvature flow.

TheOREM 2.1. Gradient descent of arc length with respect to the following inner product

$$
\langle f, g\rangle_{c}=\int_{0}^{1} f g \sqrt{x_{\alpha}^{2}+y_{\alpha}^{2}} d \alpha
$$

gives rise to motion by curvature.

The proof is relatively straightforward and will not be given here.

2.2. Level set formulation. In the level set method, the interface $\Gamma$ is represented implicitly as the zero level set of a Lipschitz function which we will denote by $\phi$; i.e., we have

$$
\Gamma=\{\boldsymbol{x}: \phi(\boldsymbol{x}, t)=0\} .
$$

If the normal speed of the interface is $v_{n}$, then the time evolution of $\phi$ is given by

$$
\frac{\partial \phi}{\partial t}+v_{n}|\nabla \phi|=0
$$

Let us now consider the case when the normal velocity is $\kappa$, where $\kappa$ is the mean curvature. We may easily write the mean curvature in terms of $\phi$ as

$$
\kappa=-\nabla \cdot\left(\frac{\nabla \phi}{|\nabla \phi|}\right) .
$$

Therefore, the level set equation for motion by mean curvature is

$$
\frac{\partial \phi}{\partial t}-\nabla \cdot\left(\frac{\nabla \phi}{|\nabla \phi|}\right)|\nabla \phi|=0 .
$$

It is natural to ask, especially in view of the previous discussion, whether or not (2.4) can be viewed as gradient descent. One formulation in this direction is due to Zhao et al. $[23,24]$, where they write the arc length of $\Gamma$ as

$$
L_{0}(\phi)=\int \delta(\phi)|\nabla \phi| d \boldsymbol{x} .
$$

They then consider the Fréchet derivative of $L_{0}$ with respect to the $L^{2}$ inner product to obtain

$$
\frac{\delta L_{0}}{\delta \phi}=-\delta(\phi) \nabla \cdot\left(\frac{\nabla \phi}{|\nabla \phi|}\right)
$$


This is quite close to what we want. In fact, if one replaces $\delta(\phi)$ by $|\nabla \phi|$, as is done by Zhao et al. [23, 24], then one would have a gradient descent formulation for curvature flow using a level set representation.

To achieve our objective we must make two observations. The first is that the evolution equation for $\phi$ given by (2.4) involves all the level curves, whereas the expression for arc length given by (2.5) singles out the zero level set. To incorporate all the arc length level sets in a single functional we first express the arc length of the $\beta$-th level set as

$$
L_{\beta}(\phi)=\int \delta(\phi-\beta)|\nabla \phi| d \boldsymbol{x}
$$

A measure of the total length of all the level curves is then

$$
L(\phi)=\int L_{\beta}(\phi) d \beta=\int|\nabla \phi| d \boldsymbol{x} .
$$

Equation (2.8) is the familiar coarea formula for the total variation of functions. Taking the Fréchet derivative of (2.8) with respect to the $L^{2}$ inner product, we get

$$
\frac{\delta L}{\delta \phi}=-\nabla \cdot\left(\frac{\nabla \phi}{|\nabla \phi|}\right) .
$$

The reason that we do not obtain curvature flow constitutes our second observation: When one takes variations with respect to $\phi$ this includes variations that do not move the level sets, namely those that change only $\nabla \phi$. To circumvent this problem we note that, for $\epsilon$ sufficiently small, $\phi+\varepsilon u|\nabla \phi|$ will perturb the locations of the level set by an amount given by $\varepsilon u$. In other words, if $\Gamma_{\beta}=\{\boldsymbol{x}: \phi=\beta\}$, then the level curves of $\{\boldsymbol{x}: \phi+\varepsilon u|\nabla \phi|=\beta\}$ will be, approximately, a distance of $\varepsilon u$ from $\Gamma_{\beta}$. It is natural then to consider the following computation:

$$
\lim _{\varepsilon \rightarrow 0} \frac{d}{d \varepsilon} L(\phi+\varepsilon u|\nabla \phi|)=-\int_{\Omega} \nabla \cdot\left(\frac{\nabla \phi}{|\nabla \phi|}\right)|\nabla \phi| u d \boldsymbol{x} .
$$

Therefore, if we use the above expression to define the Fréchet derivative, then we find that motion by mean curvature is gradient descent of (2.8). An equivalent interpretation of the computation given above is the following:

THEOREM 2.2. Level set motion of curvature flow is gradient decent of the total variation of $\phi$ with respect to the inner product, denoted the level set inner product, $\langle u, v\rangle=\int_{\Omega} \frac{u v}{|\nabla \phi|} d \boldsymbol{x}$.

This result is known $[5,6,8]$. The purpose of the preceding discussion is to establish a connection between the variational approach of Zhao et al. [23, 24] and that found in $[5,6,8]$.

\section{Multi-phase motion with interfacial and bulk energies}

In this section, we focus on the problem of $n$ phases that partition a domain $\Omega$. We will denote the region occupied by the $i$-th phase $\Sigma_{i} \cdot \operatorname{Vol}\left(\Sigma_{i}\right)$ and $\operatorname{Per}\left(\Sigma_{i}\right)$ will denote its volume and perimeter (surface area); in the plane, these would be the area of $\Sigma_{i}$ and the length of the curve $\partial \Sigma_{i}$, respectively. Any given point in $\Omega$ belongs to one and only one of the phases; more precisely, we have the following requirements:

$$
\bigcup_{i=1}^{n} \Sigma_{i}=\Omega \text { and } \operatorname{Vol}\left(\Sigma_{i} \cap \Sigma_{j}\right)=0 \text { whenever } i \neq j \text {. }
$$


The constant $e_{i} \geq 0$ will denote the "bulk energy density" of the $i$-th phase, and the constant $\sigma_{i} \geq 0$ its "surface energy density". The total energy of the configuration $\Sigma_{1}, \ldots, \Sigma_{n}$ is given by

$$
E=\sum_{i=1}^{n} \sigma_{i} \operatorname{Per}\left(\Sigma_{i}\right)+e_{i} \operatorname{Vol}\left(\Sigma_{i}\right)
$$

We remark that systems described by (1.2) are more general than the ones we are now considering; in particular, not all choices of surface tensions $f_{i, j}$ can be described by a choice of the $\sigma_{i}$. A very important point is that the energy (3.2) is lower semicontinuous with respect to its variables $\Sigma_{i}$. It is not clear whether or not the remaining systems are amenable to our formulation.

We shall let $\boldsymbol{n}_{i}$ denote the unit inward-directed normal to the boundary $\partial \Sigma_{i}$ of the $i$-th phase. If we consider gradient descent of (3.2), then the normal velocity of the boundary $\partial \Sigma_{i}$ of phase $i$, which is adjacent to another phase, say phase $j$, is

$$
v_{i j}=e_{i}-e_{j}+\left(\sigma_{i}+\sigma_{j}\right) \kappa_{i j}
$$

where $\kappa_{i j}$ is the curvature of the interface $\partial \Sigma_{i} \cap \partial \Sigma_{j}$. Notice that as we mentioned above, this is more restrictive than (1.1) since $f_{i j}$ must take the form $\sigma_{i}+\sigma_{j}$.

Our goal is to develop a variational formulation for this problem in terms of level set functions describing the phases. The first work in this direction is due to Zhao et al. $[23,24]$. In their approach, a level set function $\phi_{i}$ is introduced for each phase, so that the points belonging to that phase are given by the set

$$
\Sigma_{i}=\left\{\boldsymbol{x}: \phi_{i}(\boldsymbol{x})>0\right\} .
$$

In order to ensure that these sets form a partition of the domain $\Omega$, it is then necessary to impose explicitly the conditions that these sets be disjoint, and that they cover up $\Omega$ completely (i.e., conditions (3.1)). In terms of the level set functions $\phi_{i}$, these requirements can be viewed as the following pointwise constraint:

$$
\sum_{i} H\left(\phi_{i}(\boldsymbol{x})\right)=1
$$

For technical reasons, they replaced the above constraint with

$$
\int_{\Omega}\left(\sum_{i} H\left(\phi_{i}(\boldsymbol{x})\right)-1\right)^{2} d \boldsymbol{x}=\varepsilon \ll 1 .
$$

The authors then use standard level set methods to cast (3.2) in terms of level set functions. The constraint is handled by introducing a Lagrange multiplier $\lambda$. Zhao et al. $[23,24]$ then consider gradient descent with respect to the $L^{2}$ inner product. After replacing the resulting Dirac delta functions by $\left|\nabla \phi_{i}\right| \mathrm{s}$, the authors show that the normal speed of the interface of the $i$-th phase is

$$
v_{i}=e_{i}+\sigma_{i} \kappa_{i}-\lambda \Delta A,
$$

where

$$
\begin{aligned}
& \Delta A=\text { total amount of overlap among all regions } \\
& - \text { amount of vacuum between all regions }
\end{aligned}
$$


and $\lambda$ is the Lagrange multiplier (see (2.16) in [24], but note that (2.16) has a sign mistake). It is not clear that (3.5) will ever agree with (3.3). In the case $n=2$ with $\sigma_{1}=\sigma_{2}=0$, one can apply an argument similar to that found in [25] to show $\lambda \Delta A \approx \frac{1}{2}\left(e_{1}+e_{2}\right)$, which combined with (3.5) shows that $v_{1}=-v_{2}=\frac{1}{2}\left(e_{1}-e_{2}\right)$, which is half the expected amount. It should be emphasized that this argument is based on uncontrolled approximations which would be difficult to make rigorous. Nevertheless, this seems to agree quite closely with the numerical results presented in Figure 5 of $[24]$.

Thus it would appear that the introduction of the no overlapping/no vacuum constraint will affect the dynamics. In general it is not easy to determine in what way the dynamics will deviate from (3.3). Moreover, the presence of the constraint might contribute to the stiffness of the problem. Therefore it seems it would be useful to have a level set description that has no constraints for this problem. This is done in the next section.

3.1. Unconstrained formulation. In this section, we propose an alternative level set based formulation that does not require explicit constraints for the multiphase flow problem: instead, the constraints will be satisfied automatically by our construction. The idea is similar to, but different from, the multiphase level set representation used in [22] for the numerical treatment of the piecewise constant Mumford-Shah image segmentation model. Our formulation is closest to the level set based approach [26] to another vision problem, namely the 2.1D sketch model $[11,12]$ of Nitzberg, Mumford, and Shiota. In this model, a scene consisting of planar objects which may be occluding each other is to be represented. The objects are assumed to be ordered according to their distances from the observer, so that those closer to the observer can occlude those further away. In [26], this is accomplished by describing the space occupied by each such planar object by a level set function. Since the model allows occlusions, these sets are not required to be disjoint. However, one defines the visible part of any object in the scene, which is the part of the object that is not occluded by those in front of it. The visible parts of objects are pairwise disjoint, and constitute a partitioning of the domain.

Our approach is to use this representation of a partitioning of the domain by sets that are allowed to overlap. The essential difference of this representation from the one in [22] is the following: In order for the representation in [22] to describe, for example, three regions, one of the phases needs to become empty, leading to the degenerate situation where boundaries of two separate phases completely overlap. This prevents the technique from faithfully simulating, for instance, the motion by curvature of a triple junction. As is pointed out in [13], for example the $120^{\circ}$ condition can be violated by this technique (which is not a drawback and is in fact a possible advantage for the original purpose of this method - image segmentation). It is pointed out in [13] that if one wants to faithfully simulate motion of, say, a triple junction under motion by curvature, one can resort to the Lagrange multiplier based approach in [24] rather than the unconstrained method of [22]. We will show below that one can faithfully simulate these motions with an unconstrained formulation.

Although our representation is closely related to that of $[7,26]$, there is a significant difference. In the model dealt with by these previous works, the motion of the interfaces is derived from an interfacial energy that involves the entire boundaries of the regions (whereas the bulk energy terms involve the visible parts of the phases). In the more standard multi-phase problems we are dealing with in this work, overlapping parts of phases do not make any sense, and the interfacial energies involve only the 
visible parts of the boundaries (and also the bulk energy terms involve only the visible parts of the regions).

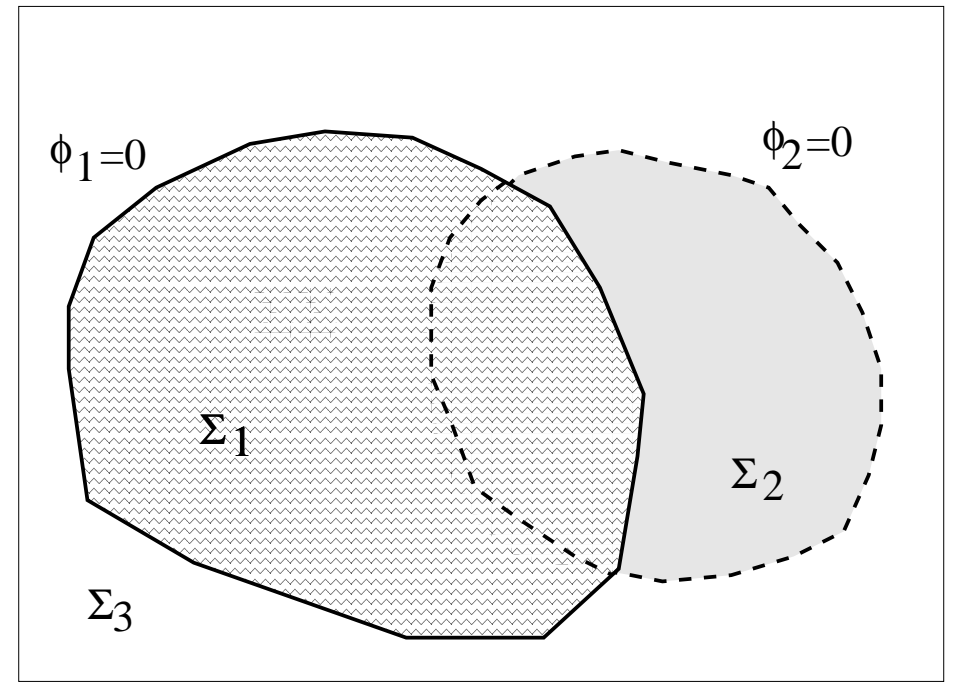

FIG. 3.1. The setup for three phases using our approach. The region with the jagged lines is $\Sigma_{1}$, the gray region is $\Sigma_{2}$, and the white region is $\Sigma_{3}$. The solid line marks $\phi_{1}=0$, and $\phi_{1}>0$ in the region surrounded by the solid line. The dotted line shows where $\phi_{2}=0$. In the region surrounded by the dotted line, $\phi_{2}>0$.

We shall now describe the level set representation for the unconstrained formulation in which $(n-1)$ level set functions are utilized to describe partitions of the domain into $n$ different phases. Recall that we use $\Sigma_{i}$ to denote the region occupied by phase $i$. We begin with the simplest case, $n=2$. Here, we have

$$
\Sigma_{1}=\left\{\boldsymbol{x}: \phi_{1}>0\right\}
$$

and

$$
\Sigma_{2}=\left\{\boldsymbol{x}: \phi_{1}<0\right\} .
$$

The first non-trivial case is $n=3$ (see Figure 1 ). In this case, we have

$$
\begin{gathered}
\Sigma_{1}=\left\{\boldsymbol{x}: \phi_{1}>0\right\}, \\
\Sigma_{2}=\left\{\boldsymbol{x}: \phi_{1}<0\right\} \cap\left\{\boldsymbol{x}: \phi_{2}>0\right\}=\left\{\boldsymbol{x}: \min \left(-\phi_{1}, \phi_{2}\right)>0\right\}, \\
\Sigma_{3}=\left\{\boldsymbol{x}: \phi_{1}<0\right\} \cap\left\{\boldsymbol{x}: \phi_{2}<0\right\}=\left\{\boldsymbol{x}: \min \left(-\phi_{1},-\phi_{2}\right)>0\right\} .
\end{gathered}
$$

It is easy to check that these sets do not overlap.

For the general case, we first introduce the following notation

$$
\begin{aligned}
& M_{1}\left(\xi_{1}, \ldots, \xi_{n-1}\right)=\xi_{1}, \\
& M_{i}\left(\xi_{1}, \ldots, \xi_{n-1}\right)=\min \left\{\xi_{i}, \min _{j=1, \ldots, i-1}-\xi_{j}\right\} \text { for } i=2, \ldots, n-1, \\
& M_{n}\left(\xi_{1}, \ldots, \xi_{n-1}\right)=\min \left\{-\xi_{1}, \ldots,-\xi_{n-1}\right\},
\end{aligned}
$$


and then the definition

$$
\psi_{i}(\boldsymbol{x})=M_{i}\left(\phi_{1}(\boldsymbol{x}), \ldots, \phi_{n-1}(\boldsymbol{x})\right) \quad \text { for } \quad i=1, \ldots, n \text {. }
$$

Our representation for an $n$-phase partition is

$$
\Sigma_{i}=\left\{\boldsymbol{x}: \psi_{i}>0\right\} \quad \text { for } \quad i=1, \ldots, n .
$$

These automatically satisfy the nonoverlap constraint, namely,

1. $\Sigma_{i} \cap \Sigma_{j}=\emptyset$ whenever $i \neq j$, and

2. $\cup_{i} \Sigma_{i}=\Omega$.

Finally we remark that if we consider the sets

$$
\Sigma_{i}^{\prime}=\left\{\boldsymbol{x}: \phi_{i}(\boldsymbol{x})>0\right\} \quad \text { for } \quad i=1, \ldots, n-1,
$$

one can then view the sets $\Sigma_{i}$ as the "visible" parts of $\Sigma_{i}^{\prime}$ (i.e. parts of $\Sigma_{i}^{\prime}$ unoccluded by other sets). The relation between $\Sigma_{i}$ and $\Sigma_{i}^{\prime}$ is given by

$$
\Sigma_{i}:=\Sigma_{i}^{\prime} \backslash \bigcup_{j=1}^{i-1} \Sigma_{j}^{\prime}
$$

with the understanding that $\Sigma_{1}=\Sigma_{1}^{\prime}$.

3.2. Variational level set formulation. In this section we describe the variational formulation according to the representation of multiple phases described in the previous section. To clarify the basic idea, we first consider the case $n=2$. Here, $\Sigma_{1}=\left\{\boldsymbol{x}: \phi_{1}>0\right\}$ and $\Sigma_{2}=\left\{\boldsymbol{x}: \phi_{1}<0\right\}$. Therefore the total energy of the system is

$$
E_{0}=\int_{\Omega}\left[\sigma \delta\left(\phi_{1}\right)\left|\nabla \phi_{1}\right|+e_{1} H\left(\phi_{1}\right)+e_{2}\left(1-H\left(\phi_{1}\right)\right)\right] d \boldsymbol{x},
$$

where $\sigma=\sigma_{1}+\sigma_{2}$. However, gradient descent of this energy will give rise to delta functions. To remove this difficulty, we apply a similar strategy as in Section 2.2: the energy is modified so that all the other level curves behave in a similar way. To that end, we consider the following energy associated to the $\beta$-th level curve

$$
E_{\beta}=\int\left[\sigma \delta\left(\phi_{1}-\beta\right)\left|\nabla \phi_{1}\right|+e_{1} H\left(\phi_{1}-\beta\right)+e_{2}\left(1-H\left(\phi_{1}-\beta\right)\right)\right] d \boldsymbol{x} .
$$

Now we include energy of all the level curves:

$$
\begin{aligned}
E & =\int E_{\beta} d \beta \\
& =\int_{\Omega}\left|\nabla \phi_{1}\right| d \boldsymbol{x}+\int_{\beta} \int_{\Omega}\left[e_{1} H\left(\phi_{1}-\beta\right)+e_{2}\left(1-H\left(\phi_{1}-\beta\right)\right)\right] d \boldsymbol{x} d \beta .
\end{aligned}
$$

The Fréchet derivative of $E$ with respect to the level set inner product gives

$$
\frac{\delta E}{\delta \phi_{1}}=\left[-\sigma \nabla \cdot\left(\frac{\nabla \phi_{1}}{\left|\nabla \phi_{1}\right|}\right)+e_{1}-e_{2}\right]\left|\nabla \phi_{1}\right| .
$$

Therefore, gradient descent with respect to the level set inner product yields the following evolution:

$$
\frac{\partial \phi_{1}}{\partial t}+\left[e_{1}-e_{2}-\sigma \nabla \cdot\left(\frac{\nabla \phi_{1}}{\left|\nabla \phi_{1}\right|}\right)\right]\left|\nabla \phi_{1}\right|=0
$$


We recall that $\kappa_{12}=-\nabla \cdot \boldsymbol{n}_{12}$ where $\boldsymbol{n}_{12}$ is the unit normal into phase 1 from phase 2 . In this case $\boldsymbol{n}_{12}=\nabla \phi_{1} /\left|\nabla \phi_{1}\right|$, so it follows that the normal speed of phase 1 is then

$$
v_{1}=e_{1}-e_{2}+\left(\sigma_{1}+\sigma_{2}\right) \kappa_{12}
$$

as expected.

3.3. General case. We now present the derivation of our level set method for multiphase motion in the general case. The total energy associated to the zero level set is now given by

$$
E_{0}\left(\phi_{1}, \ldots, \phi_{n-1}\right)=\sum_{i=1}^{n} \int_{\Omega}\left(\sigma_{i} \delta\left(\psi_{i}\right)\left|\nabla \psi_{i}\right|+e_{i} H\left(\psi_{i}\right)\right) d \boldsymbol{x} .
$$

As before we consider the total energy associated to the $\beta$-th level set, which is

$$
E_{\beta}\left(\phi_{1}, \ldots, \phi_{n-1}\right)=\sum_{i=1}^{n} \int_{\Omega}\left(\sigma_{i} \delta\left(\psi_{i}-\beta\right)\left|\nabla \psi_{i}\right|+e_{i} H\left(\psi_{i}-\beta\right)\right) d \boldsymbol{x},
$$

and the total energy of the all the level sets is then

$$
\begin{aligned}
E\left(\phi_{1}, \ldots, \phi_{n-1}\right) & =\int E_{\beta}\left(\phi_{1}, \ldots, \phi_{n-1}\right) d \beta \\
& =\sum_{i=1}^{n} \int_{\Omega}\left(\sigma_{i}\left|\nabla \psi_{i}\right|+e_{i} \int_{\beta} H\left(\psi_{i}-\beta\right) d \beta\right) d \boldsymbol{x} .
\end{aligned}
$$

The level set inner product for multiple level sets is a straightforward extension of the scalar case. If we let $\boldsymbol{u}$ and $\boldsymbol{v}$ be $n-1$ dimensional vector-valued functions of $\boldsymbol{x}$ then the level set inner product for $n$ phases is

$$
\langle\boldsymbol{u}, \boldsymbol{v}\rangle=\int_{\Omega} \boldsymbol{u}^{T} \operatorname{diag}\left(\left|\nabla \phi_{1}\right|^{-1}, \ldots,\left|\nabla \phi_{n-1}\right|^{-1}\right) \boldsymbol{v} d \boldsymbol{x} .
$$

Gradient flow for $E$ with respect to the level set inner product turns out to be

$$
\partial_{t} \phi_{i}=\left|\nabla \phi_{i}\right| \sum_{j=i}^{n} \frac{\partial \psi_{j}}{\partial \phi_{i}}\left[\sigma_{j} \nabla \cdot\left(\frac{\nabla \psi_{j}}{\left|\nabla \psi_{j}\right|}\right)-e_{j}\right]
$$

where we have made use of the fact that

$$
\frac{\partial \psi_{j}}{\partial \phi_{i}}=0 \text { for } i>j
$$

In addition, there are explicit expressions for the other terms. For $j=1$ we have

$$
\frac{\partial \psi_{1}}{\partial \phi_{i}}=\delta_{1 i}
$$

where $\delta_{i j}$ is the Kronecker delta function. For $j=2$ to $n-1$ we have

$$
\frac{\partial \psi_{j}}{\partial \phi_{i}}=\left\{\begin{array}{c}
-H\left(\phi_{i}+\phi_{j}\right) \prod_{\substack{k=1, k \neq i \\
j-1}}^{j-1} H\left(\phi_{i}-\phi_{k}\right) \quad \text { for } \quad i<j \\
\prod_{k=1} H\left(-\phi_{j}-\phi_{k}\right) \text { for } i=j
\end{array}\right.
$$


and for $j=n$ we have

$$
\frac{\partial \psi_{n}}{\partial \phi_{i}}=-\prod_{k=1, k \neq i}^{n-1} H\left(\phi_{i}-\phi_{k}\right) .
$$

3.3.1. Explicit example for $\mathbf{n}=\mathbf{3}$. We can either use the above formulas or note that $\psi_{1}=\phi_{1}, \psi_{2}=\min \left(\phi_{2},-\phi_{1}\right)$, and $\psi_{3}=\min \left(-\phi_{1},-\phi_{2}\right)$. In either case one finds

$$
\begin{aligned}
& \frac{\partial \psi_{1}}{\partial \phi_{i}}=\left\{\begin{array}{lll}
1 & \text { for } & i=1 \\
0 & \text { for } & i=2,
\end{array}\right. \\
& \frac{\partial \psi_{2}}{\partial \phi_{i}}=\left\{\begin{array}{rll}
-H\left(\phi_{1}+\phi_{2}\right) & \text { for } & i=1 \\
H\left(-\phi_{1}-\phi_{2}\right) & \text { for } & i=2,
\end{array}\right.
\end{aligned}
$$

and

$$
\frac{\partial \psi_{3}}{\partial \phi_{i}}=\left\{\begin{array}{lll}
-H\left(\phi_{1}-\phi_{2}\right) & \text { for } & i=1 \\
-H\left(-\phi_{1}+\phi_{2}\right) & \text { for } & i=2
\end{array}\right.
$$

The gradient descent equations for the level set functions $\phi_{1}, \phi_{2}$ are

$$
\begin{aligned}
\partial_{t} \phi_{1}=\left|\nabla \phi_{1}\right|\left(\left[-e_{1}+\sigma_{1} \nabla \cdot\left(\frac{\nabla \phi_{1}}{\left|\nabla \phi_{1}\right|}\right)\right]\right. & \\
-H\left(\phi_{1}+\phi_{2}\right) & {\left[-e_{2}+\sigma_{2} \nabla \cdot\left(\frac{\nabla \min \left(\phi_{2},-\phi_{1}\right)}{\left|\nabla \min \left(\phi_{2},-\phi_{1}\right)\right|}\right)\right] } \\
& \left.-H\left(\phi_{1}-\phi_{2}\right)\left[-e_{3}+\sigma_{3} \nabla \cdot\left(\frac{\nabla \min \left(-\phi_{1},-\phi_{2}\right)}{\left|\nabla \min \left(-\phi_{1},-\phi_{2}\right)\right|}\right)\right]\right)
\end{aligned}
$$

and

$$
\begin{aligned}
\partial \phi_{2}=\left|\nabla \phi_{2}\right|\left(-H\left(-\phi_{1}-\phi_{2}\right)\right. & {\left[-e_{2}+\sigma_{2} \nabla \cdot\left(\frac{\nabla \min \left(\phi_{2},-\phi_{1}\right)}{\left|\nabla \min \left(\phi_{2},-\phi_{1}\right)\right|}\right)\right] } \\
& \left.-H\left(-\phi_{1}+\phi_{2}\right)\left[-e_{3}+\sigma_{3} \nabla \cdot\left(\frac{\nabla \min \left(-\phi_{2},-\phi_{1}\right)}{\left|\nabla \min \left(-\phi_{2},-\phi_{1}\right)\right|}\right)\right]\right) .
\end{aligned}
$$

Note that when $\phi_{1} \approx 0$ and $\phi_{2}<0$, one is in the vicinity of the interface between $\Sigma_{1}$ and $\Sigma_{3}$ (i.e. $\left.\Gamma_{13}\right)$. In this case, $H\left(\phi_{1}+\phi_{2}\right)=0$ and $H\left(\phi_{1}-\phi_{2}\right)=1$. In addition, $\min \left(-\phi_{1},-\phi_{2}\right)=-\phi_{1}$. Therefore, in the region close to $\Gamma_{13}$, the evolution equation for $\phi_{1}$ is

$$
\partial_{t} \phi_{1}+\left|\nabla \phi_{1}\right|\left(e_{1}-e_{3}-\left(\sigma_{1}+\sigma_{3}\right) \nabla \cdot\left(\frac{\nabla \phi_{1}}{\left|\nabla \phi_{1}\right|}\right)\right)=0
$$

As a consequence one finds that

$$
v_{13}=e_{1}-e_{3}+\left(\sigma_{1}+\sigma_{3}\right) \kappa_{13}
$$

as expected. A similar calculation will show the other normal speeds are in agreement with (3.3). 
REMARK 3.1. A possible alternative to the representation proposed and utilized in this paper can be based on the idea in [3]. There, $n$ possibly overlapping subsets $\Sigma_{1}^{\prime}, \ldots, \Sigma_{n}^{\prime}$ of the domain $\Omega$ are used to partition $\Omega$ into $2^{n}$ disjoint components given by:

$$
\Sigma_{v}=\left(\bigcap_{j: v(j)=1} \Sigma_{j}^{\prime}\right) \cap\left(\bigcap_{j: v(j)=0}\left(\Sigma_{j}^{\prime}\right)^{c}\right),
$$

where $v$ is a mapping $v:\{1,2, \ldots, n\} \rightarrow\{0,1\}$. With this representation, energy (3.2) can be written:

$$
E=\sum_{\{v:\{1, \ldots, n\} \rightarrow\{0,1\}\}} \sigma_{v} \operatorname{Per}\left(\Sigma_{v}\right)+e_{v} \operatorname{Vol}\left(\Sigma_{v}\right)
$$

Note that this is not what is done in [3]; rather the authors of [3] replace the surface energy terms by perimeters of the sets $\Sigma_{j}^{\prime}$ instead, which does not yield energy (3.2); nevertheless, as mentioned earlier, this can be advantageous for image processing applications.

This alternative approach has been implemented using a level set formulation. Preliminary results seem to suggest that the resulting flow does not work, possibly because it is significantly more stiff than the formulation given in Section 3.1. However, given the potential benefits of this representation, it seems worth further exploration.

3.4. Almgren-Taylor-Wang approximating flows. In this section, we show how the technique described in sections 3.1 and 3.2 can be used to solve via level sets a variational formulation of multi-phase motion that is motivated by an idea of Almgren, Taylor, and Wang. In [1], these authors introduced an algorithm for approximating at discrete times the motion by mean curvature of an interface. Their algorithm consists in solving a variational problem at every time step. Generalization of their method to the motion of multiple phases is discussed in [20, 21, 4]. The formulation in [21], although probably more principled and accurate than others as explained in that paper, seems to be computationally very difficult. Here we, therefore, consider a simplified formulation that seems equivalent to the one in [4]. For the sake of clarity, we will illustrate it in the case of three-phase motion; its generalization to more than three phases will be quite clear.

The main idea is to solve the following variational problem in order to advance the solution $\left(\Sigma_{1}^{n}, \Sigma_{2}^{n}, \Sigma_{3}^{n}\right)$ at the $n$-th time step $t=n \delta t$ to the solution $\left(\Sigma_{1}^{n+1}, \Sigma_{2}^{n+1}, \Sigma_{3}^{n+1}\right)$ at the next time step:

$$
\min _{\substack{\Sigma_{1}, \Sigma_{2}, \Sigma_{3}, \Sigma_{i} \cap \Sigma_{j}=\emptyset \forall i \neq j, j=1 \\ \bigcup_{j} \Sigma_{j}=\Omega}} \sum_{j=1}^{3}\left\{\sigma_{j} \operatorname{Per}\left(\Sigma_{j}\right)+e_{j} \operatorname{Vol}\left(\Sigma_{j}\right)+\frac{1}{\delta t} \int_{\Sigma_{j} \Delta \Sigma_{j}^{n}}\left|d_{\Sigma_{j}^{n}(\boldsymbol{x})}\right| d \boldsymbol{x}\right\}
$$

where $d_{\Sigma_{j}^{n}}(\boldsymbol{x})$ is the signed distance function to $\Sigma_{j}^{n}$, and $\Delta$ denotes the symmetric difference operator for two sets. The minimizing triplet of sets, $\left(\Sigma_{1}, \Sigma_{2}, \Sigma_{3}\right)$, become the solution at the $(n+1)$-th time step. Note that the only coupling between the unknown sets $\Sigma_{j}$ of the variational problem takes place through the constraint.

First, note that for two sets $A$ and $B$, we have the following:

$$
\int_{A \triangle B}\left|d_{B}\right| d \boldsymbol{x}=\int_{B} d_{B} d x-\int_{A} d_{B} d \boldsymbol{x},
$$


Since the first term on the right hand side is constant with respect to variations of $A$, we have the equivalence of the following variational problems:

$$
\min _{A} \int_{A \triangle B}\left|d_{B}\right| d \boldsymbol{x} \Longleftrightarrow \min _{A}-\int_{A} d_{B} d \boldsymbol{x} .
$$

Consequently, we can write problem (3.6) as follows:

$$
\min _{\substack{\Sigma_{1}, \Sigma_{2}, \Sigma_{3} \\ \Sigma_{i} \cap \Sigma_{j}=\emptyset \forall i \neq j \\ \cup_{j} \Sigma_{j}=\Omega}} \sum_{j=1}^{3}\left\{\sigma_{j} \operatorname{Per}\left(\Sigma_{j}\right)+e_{j} \operatorname{Vol}\left(\Sigma_{j}\right)-\frac{1}{\delta t} \int_{\Sigma_{j}} d_{\Sigma_{j}^{n}}(\boldsymbol{x}) d \boldsymbol{x}\right\} .
$$

We can apply our constraint-free level set based approach of previous sections to the solution of (3.9).

3.4.1. Level set based computation of approximating flow. We use the same level-set representation of three phases as before (Section 3.1):

$$
\begin{aligned}
& \Sigma_{1}=\left\{\boldsymbol{x}: \phi_{1}>0\right\}, \\
& \Sigma_{2}=\left\{\boldsymbol{x}: \min \left(-\phi_{1}, \phi_{2}\right)>0\right\}, \\
& \Sigma_{3}=\left\{\boldsymbol{x}: \min \left(-\phi_{1},-\phi_{2}\right)>0\right\} .
\end{aligned}
$$

The expression for the energy given by (3.9) can be expressed in terms of $\phi_{1}$ and $\phi_{2}$ in pretty much the same way as in Section 3.3; the only difference is the additional bulk energy terms in (3.9) that are responsible for limiting movement during a time step. These contribute the following terms to the total energy of all the level sets:

$$
\frac{1}{\delta t} \iint_{\Omega} \sum_{i=1}^{3} H\left(\psi_{i}-\beta\right) d_{\Sigma_{i}^{n}}(\boldsymbol{x}) d \boldsymbol{x} d \beta .
$$

where $\psi_{i}$ is given by the expressions at the top of Section 3.3.1. The resulting gradient descent equations for (3.9), given below, differ from the ones in Section 3.3 in merely the additional bulk energy terms coming from (3.10):

$$
\begin{aligned}
& \partial_{t} \phi_{1}=\left|\nabla \phi_{1}\right|\left(\left[\frac{1}{\delta t} d_{\Sigma_{1}^{n}}(\boldsymbol{x})-e_{1}+\sigma_{1} \nabla \cdot\left(\frac{\nabla \phi_{1}}{\left|\nabla \phi_{1}\right|}\right)\right]\right. \\
&-H\left(\phi_{1}+\phi_{2}\right)\left[\frac{1}{\delta t} d_{\Sigma_{2}^{n}}(\boldsymbol{x})-e_{2}+\sigma_{2} \nabla \cdot\left(\frac{\nabla \min \left(\phi_{2},-\phi_{1}\right)}{\left|\nabla \min \left(\phi_{2},-\phi_{1}\right)\right|}\right)\right] \\
&\left.\quad-H\left(\phi_{1}-\phi_{2}\right)\left[\frac{1}{\delta t} d_{\Sigma_{3}^{n}}(\boldsymbol{x})-e_{3}+\sigma_{3} \nabla \cdot\left(\frac{\nabla \min \left(-\phi_{1},-\phi_{2}\right)}{\left|\nabla \min \left(-\phi_{1},-\phi_{2}\right)\right|}\right)\right]\right)
\end{aligned}
$$

and

$$
\begin{aligned}
\partial_{t} \phi_{2}=\left|\nabla \phi_{2}\right|(- & H\left(-\phi_{1}-\phi_{2}\right)\left[\frac{1}{\delta t} d_{\Sigma_{2}^{n}}(\boldsymbol{x})-e_{2}+\sigma_{2} \nabla \cdot\left(\frac{\nabla \min \left(\phi_{2},-\phi_{1}\right)}{\left|\nabla \min \left(\phi_{2},-\phi_{1}\right)\right|}\right)\right] \\
- & \left.H\left(-\phi_{1}+\phi_{2}\right)\left[\frac{1}{\delta t} d_{\Sigma_{3}^{n}}(\boldsymbol{x})-e_{3}+\sigma_{3} \nabla \cdot\left(\frac{\nabla \min \left(-\phi_{2},-\phi_{1}\right)}{\left|\nabla \min \left(-\phi_{2},-\phi_{1}\right)\right|}\right)\right]\right) .
\end{aligned}
$$

We can think of our level sets based Almgren-Taylor-Wang formulation as providing a relaxation of our approach from the previous sections. Indeed, we have now 
introduced in essence a new time variable into our algorithm that is completely independent from the real time represented by the step size $\delta t$ that appears in the variational problem (3.6). During the solution of the variational problem of each actual time step, the interfaces thus get plenty of opportunity to adjust themselves, for example in order to attain the angle conditions (Young's law) implied by the energy. Some of our most accurate results were obtained using this approach. Indeed, we observed that the angle conditions are more accurately satisfied with this approach.

3.5. Numerical results. In this section, we present some numerical results for our unconstrained level set formulations of multiphase flow. We start with the formulations given in Section 3.3. Figure 3.2 presents the situation in which two phases have the same bulk energy density of 5 and the other is 10 . All the surface energy densities are unity. As expected the lower energy phases propagate into the high energy phase and the angles of the triple point are all approximately $120^{\circ}$. Figure 3.3 shows computations when all the bulk energies are zero and the surface energy densities are unity. In this case the motion results in curvature flow with all angles at the triple junction being $120^{\circ}$. The motion for four phases moving by curvature flow is shown in Figure 3.4. The initial condition consists of all four phases meeting at one point. This is stationary but unstable; under a small perturbation, it splits to form two triple junctions that subsequently pull apart. During these computations, the level set functions were reinitialized using the algorithm in [16] roughly between every 200 and 500 iterations of the flow, which was implemented with an explicit time discretization - the results were relatively insensitive to the exact frequency of reinitialization.

We also present several computations using the Almgren-Taylor-Wang approximating flow approach of Section 3.4, which turned out to provide some of the most accurate results we obtained. Redistancing was applied to the level set functions every now and then during these computations as well. The first computational example, shown in Figure 3.5, is the evolution of a triple junction under motion by mean curvature, with $\sigma_{j}=1$ and $e_{j}=0$ for $j=1,2,3$. The initial condition is the T-shaped data shown in the left plot. Solutions starting from this data tend toward an exact solution that consists of a constant profile that translates upwards [9]. The result of the computation at some later time is shown, and seems to be very close to the exact constant profile, with the correct angles (all $120^{\circ}$ ) at the junction. The plot on the right shows that there is indeed excellent agreement between the computed and the exact profiles.

Figure 3.6 shows the evolution starting from the same T-shaped initial data as in Figure 3.5, but this time the constants in energy (3.6) are chosen to be $\sigma_{1}=\sigma_{2}=\frac{1}{\sqrt{2}-1}$, $\sigma_{3}=1$, and $e_{1}=e_{2}=e_{3}=0$. These choices imply, via Young's law, that one $90^{\circ}$ and two $135^{\circ}$ angles should form at triple junctions. Once again, there is an exact constant profile that attracts our initial condition. The left plot of the figure shows a snapshot from the evolution, and the plot on the right compares the computed profile with the exact one. As can be seen, the error in this case is greater than in the experiment of Figure 3.5, which involved a symmetric triple junction. Nevertheless, the agreement is still very good, and compares quite favorably with e.g. phase-field based computations (such as those in [9] that present results with similar data), which are another class of methods that do not require explicit representation of interfaces and which are therefore also capable of handling topological changes.

Figure 3.7 zooms in on the junctions from the experiments of figures 3.5 and 3.6. The angles achieved by the computations are very close to what they ought to be. Note 

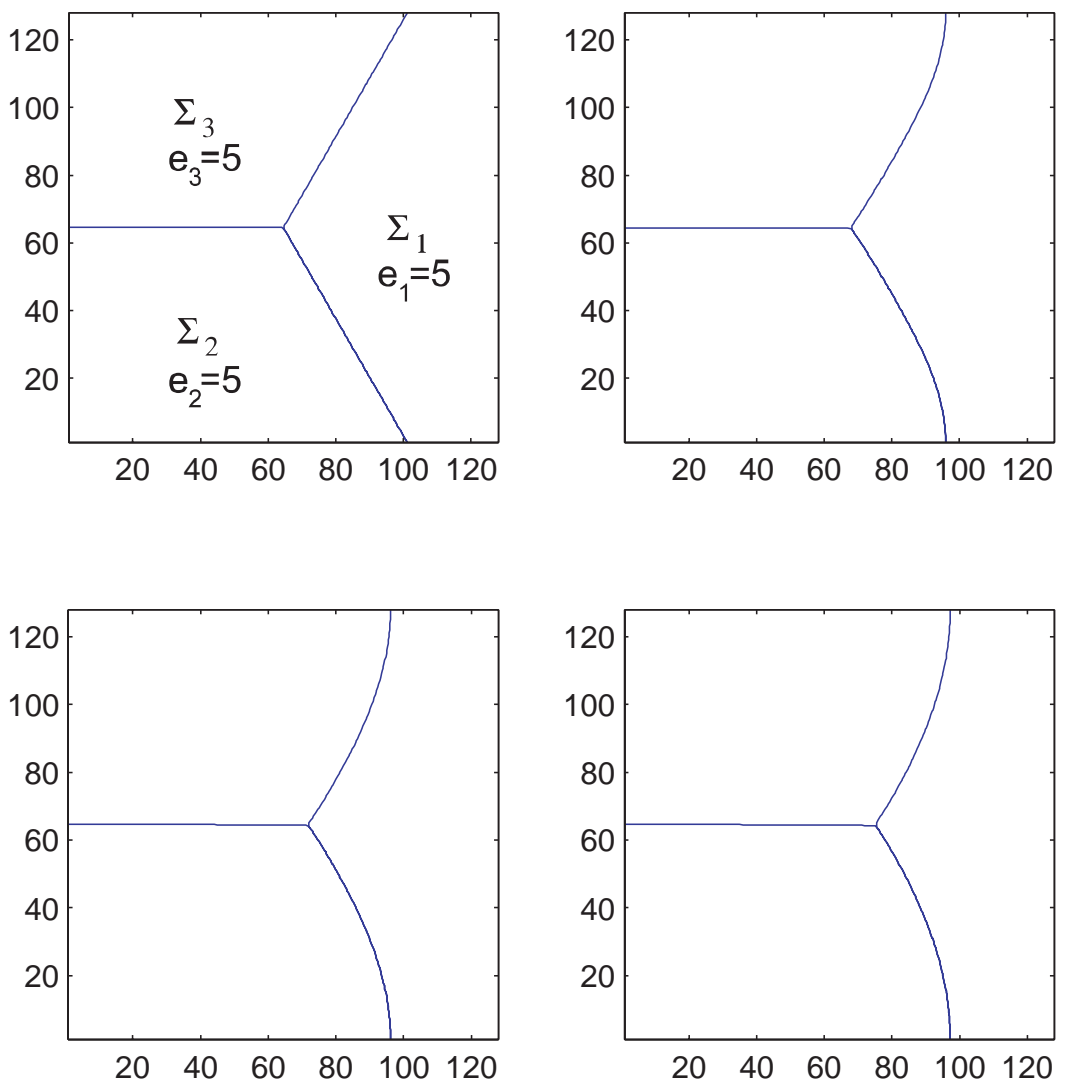

FIG. 3.2. Motion of a triple junction as gradient descent for energy (3.2) with $e_{1}=10$ (corresponding to the rightmost phase) and $e_{2}=e_{3}=5$. Evolution takes place left to right, and top to bottom. Neumann boundary conditions were imposed.

that the small (mesh sized) "triangular deviation" that appears right at the junction is merely an artifact of the contouring algorithm. Figure 3.8 shows the evolution of two initially disjoint disks under the motion generated once again by the approximating flow formulation (3.6) with $\sigma_{1}=\sigma_{2}=\sigma_{3}=1, e_{1}=e_{2}=0$, and $e_{3}=1$. The two disks represent two of the phases with 0 bulk energies; the background (complement of the disks) is the phase with bulk energy constant 1 . The disks grow at the expense of the background phase, and eventually touch. At that point, two triple junctions are formed, which subsequently pull apart, forming a new straight interface between the initially circular phases.

\section{Area preserving curvature motion}

4.1. Lagrangian formulation. Consider a curve moving in the plane. We define area preserving curvature flow

$$
\frac{d \boldsymbol{x}}{d t}=(\kappa-\langle\kappa\rangle) \boldsymbol{n},
$$



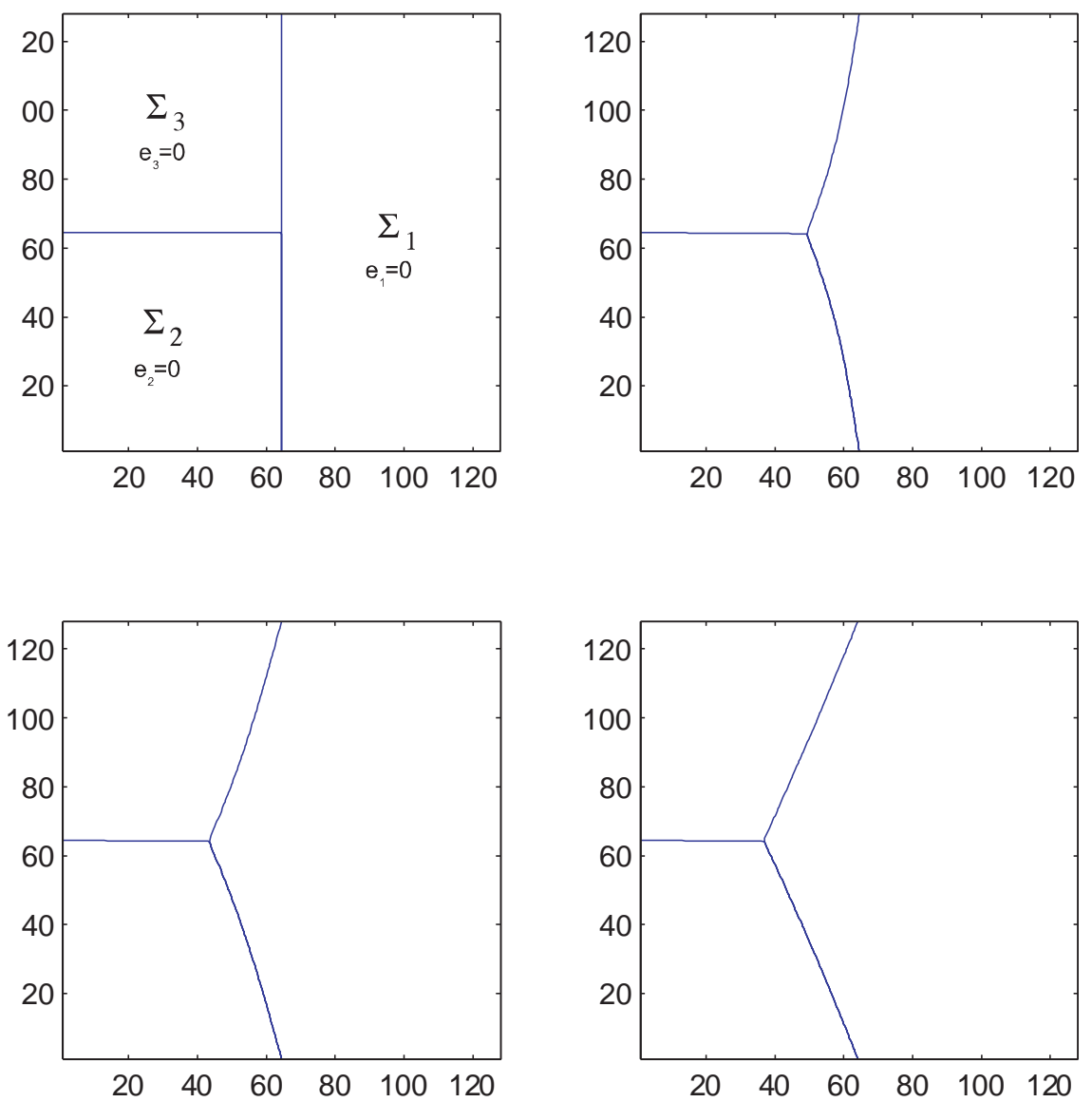

FIG. 3.3. Motion of a triple junction as gradient descent for energy (3.2) with $e_{1}=e_{2}=e_{3}=0$; hence this is motion by curvature. Dirichlet boundary conditions were imposed. The lower right hand image is essentially the stationary state.

where $\langle\kappa\rangle$ is the average curvature of the curve,

$$
\langle\kappa\rangle=\frac{\int_{0}^{1} \kappa \sqrt{x_{\alpha}^{2}+y_{\alpha}^{2}} d \alpha}{\int_{0}^{1} \sqrt{x_{\alpha}^{2}+y_{\alpha}^{2}} d \alpha} .
$$

It is easy to check that the area enclosed by such a curve remains constant under the evolution given by (4.1). We have the following theorem concerning area preserving curvature flow:

THEOREM 4.1. Area preserving curvature flow is constrained gradient descent of arc length with respect to the inner production given by (2.1).

4.2. Level set formulation. We will follow our previous approach and ask for the level set equations so that all level curves move by area preserving curvature motion. We begin with the following definition, $\langle\kappa\rangle_{\beta}$ is the average curvature of the $\beta$-th level curve. This can be expressed in terms of level set functions as 

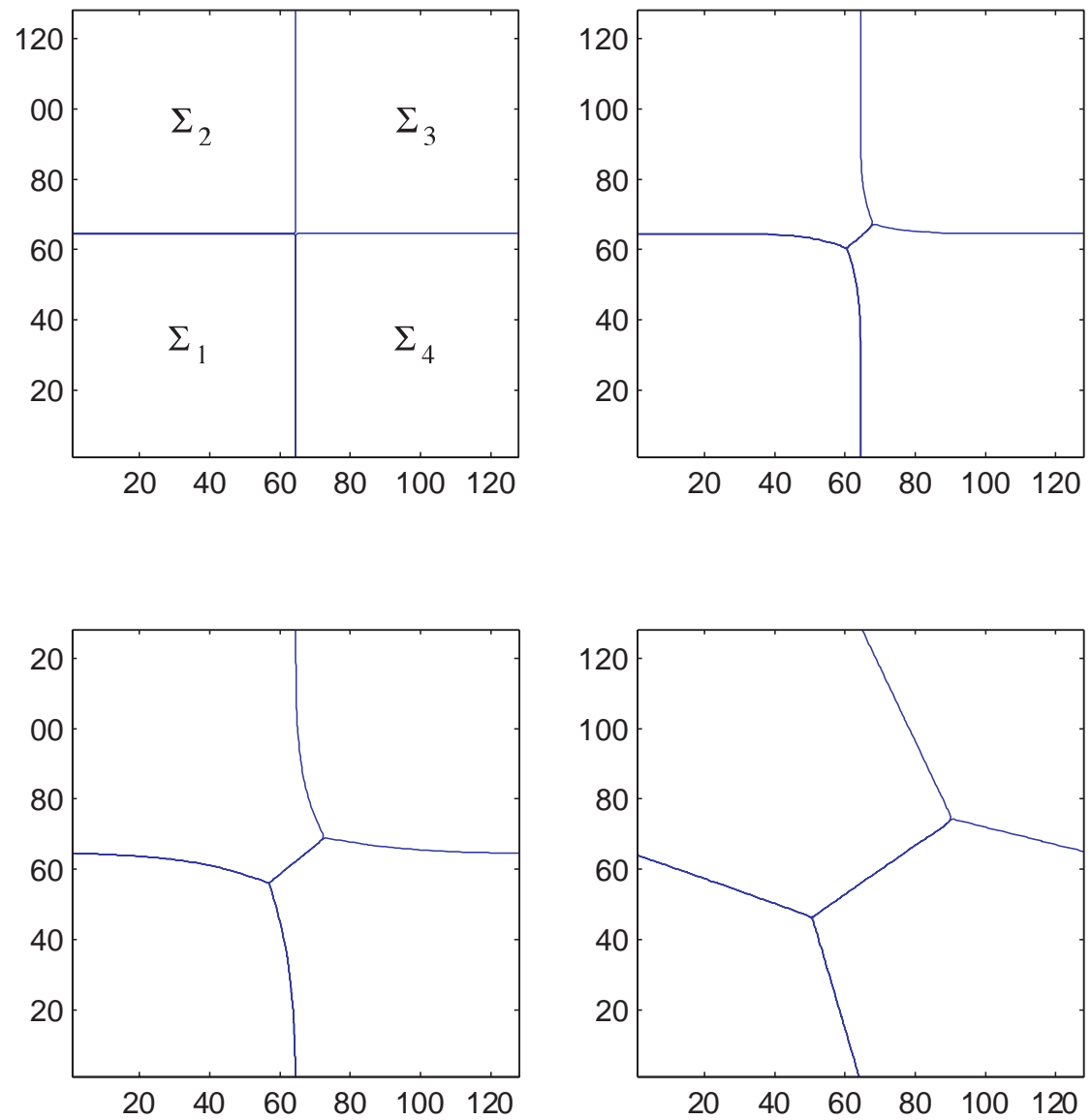

FIG. 3.4. Motion of four phases with $e_{1}=e_{2}=e_{3}=e_{4}=0$; hence this is motion by curvature. The initial condition is stationary but unstable, and splits into two triple junctions. Dirichlet boundary conditions were used.

$$
\langle\kappa\rangle_{\beta}=\frac{\int_{\Omega} \delta(\phi-\beta) \kappa|\nabla \phi| d \boldsymbol{x}}{\int_{\Omega} \delta(\phi-\beta)|\nabla \phi| d \boldsymbol{x}} .
$$

We can now write down the level set equation in which each level curve moves by area preserving mean curvature flow:

$$
\phi_{t}+\left(\kappa-\langle\kappa\rangle_{\phi}\right)|\nabla \phi|=0
$$

In order to avoid any confusion we present the following explicit expression

$$
\langle\kappa\rangle_{\phi}(\boldsymbol{x}, t)=\frac{\int_{\Omega} \delta\left(\phi\left(\boldsymbol{x}^{\prime}, t\right)-\phi(\boldsymbol{x}, t)\right) \kappa\left(\boldsymbol{x}^{\prime}, t\right)\left|\nabla \phi\left(\boldsymbol{x}^{\prime}, t\right)\right| d \boldsymbol{x}^{\prime}}{\int_{\Omega} \delta\left(\phi\left(\boldsymbol{x}^{\prime}, t\right)-\phi(\boldsymbol{x}, t)\right)\left|\nabla \phi\left(\boldsymbol{x}^{\prime}, t\right)\right| d \boldsymbol{x}^{\prime}} .
$$

We have the following Theorem concerning (4.3): 

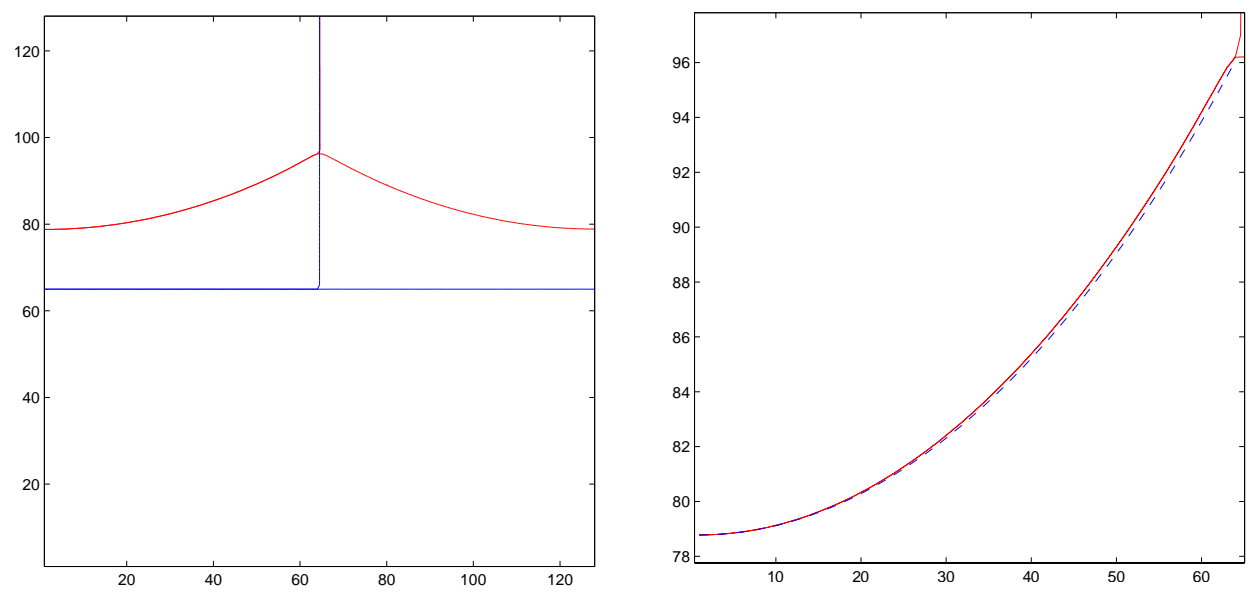

FIG. 3.5. Comparison between the exact profile (dashed curve) and the computed one (solid curve), in the case of equal surface tensions (so that $120^{\circ}$ angles are formed at the junctions) and no bulk energies. The computation was based on the formulation in Section 3.4.1.
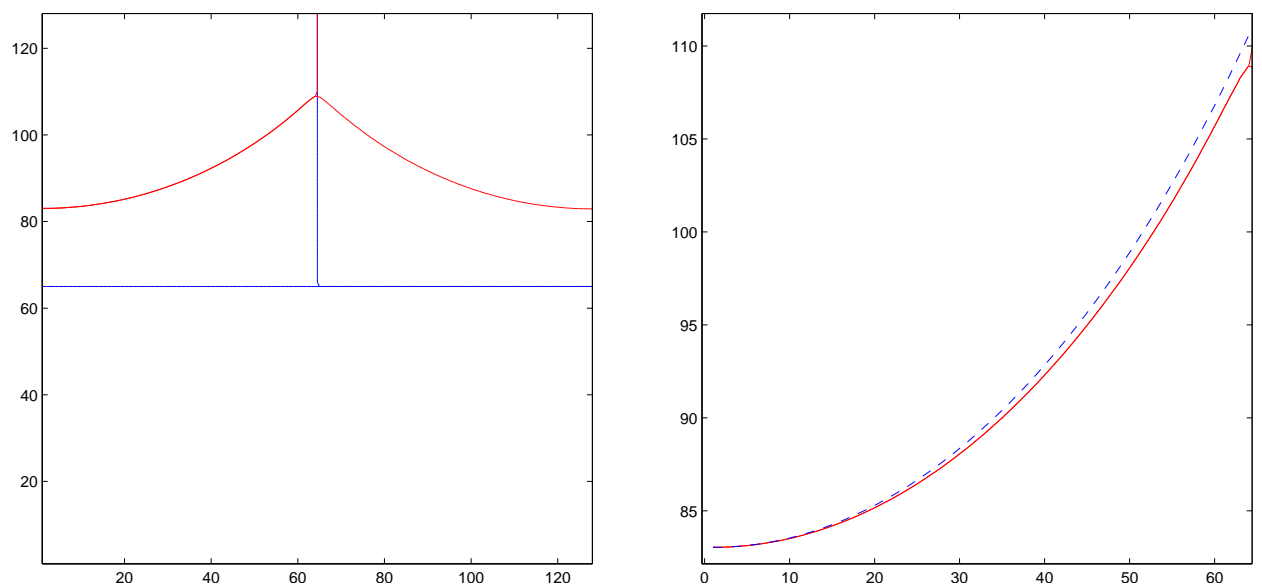

FIG. 3.6. Comparison between the exact profile (dashed curve) and the computed one (solid curve), in the case of $\sigma_{1}=\sigma_{2}=\frac{1}{\sqrt{2}-1}, \sigma_{3}=1$, and $e_{j}=0$ according to the notation in Section 3.4.1; these imply two $125^{\circ}$ and one $90^{\circ}$ angles at triple junctions. The error is larger than in the symmetric $120^{\circ}$ case shown in the previous figure, but is still very competitive with, for instance, phase-field based methods at similar resolutions (see e.g. [9]). Once again, the formulation of Section 3.4.1 was used for the computation.

THEOREM 4.2. Area preserving curvature flow is gradient descent, with respect to the level set metric, of the following,

$$
E=\int_{\Omega}|\nabla \phi| d \boldsymbol{x},
$$



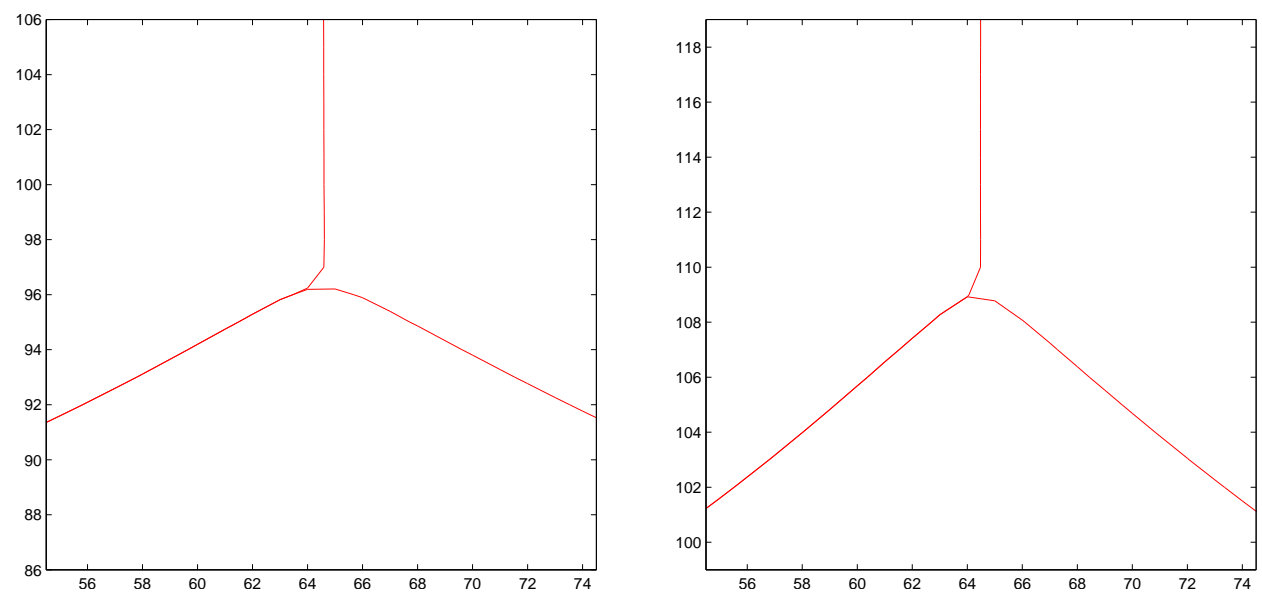

FIG. 3.7. Zoom-in on the triple junctions. The plot on the left is from the numerical experiments of Figure 3.5, where the surface tensions are equal so that a symmetric $120^{\circ}$ junction should form. the plot on the right is from the experiments of Figure 3.6, where the surface tensions imply one $90^{\circ}$ and two $135^{\circ}$ angles at a triple junction.

with the infinite set of constraints given by

$$
C_{n}=\int_{n \varepsilon-\frac{\varepsilon}{2}}^{n \varepsilon+\frac{\varepsilon}{2}} \int_{\Omega} H(-\phi+\beta) d \boldsymbol{x} d \beta
$$

in the limit $\varepsilon \rightarrow 0$, where $n \in \mathbb{Z}$.

Proof. We first observe that the Fréchet derivative of $C_{n}$ with respect to the level set inner product is

$$
\frac{\delta C_{n}}{\delta \phi}=-|\nabla \phi| \chi_{\left\{|\phi-n \varepsilon| \leq \frac{\varepsilon}{2}\right\}}
$$

where $\chi_{A}$ is the characteristic function of the the set $A$. We will now examine the problem of gradient descent of

$$
E=\int_{\Omega}|\nabla \phi| d \boldsymbol{x}
$$

subject to the constraints $C_{n}=$ constant for all $n \in Z$. Therefore we consider

$$
F=E+\sum_{n=-\infty}^{\infty} \lambda_{n} C_{n}
$$

We compute the Fréchet derivative of $F$ with respect to the level set inner product and find that its corresponding gradient descent gives the following evolution equation:

$$
\frac{\partial \phi}{\partial t}=\left[-\kappa+\sum_{n=-\infty}^{\infty} \lambda_{n} \chi_{\left\{|\phi-n \varepsilon| \leq \frac{\varepsilon}{2}\right\}}\right]|\nabla \phi| .
$$



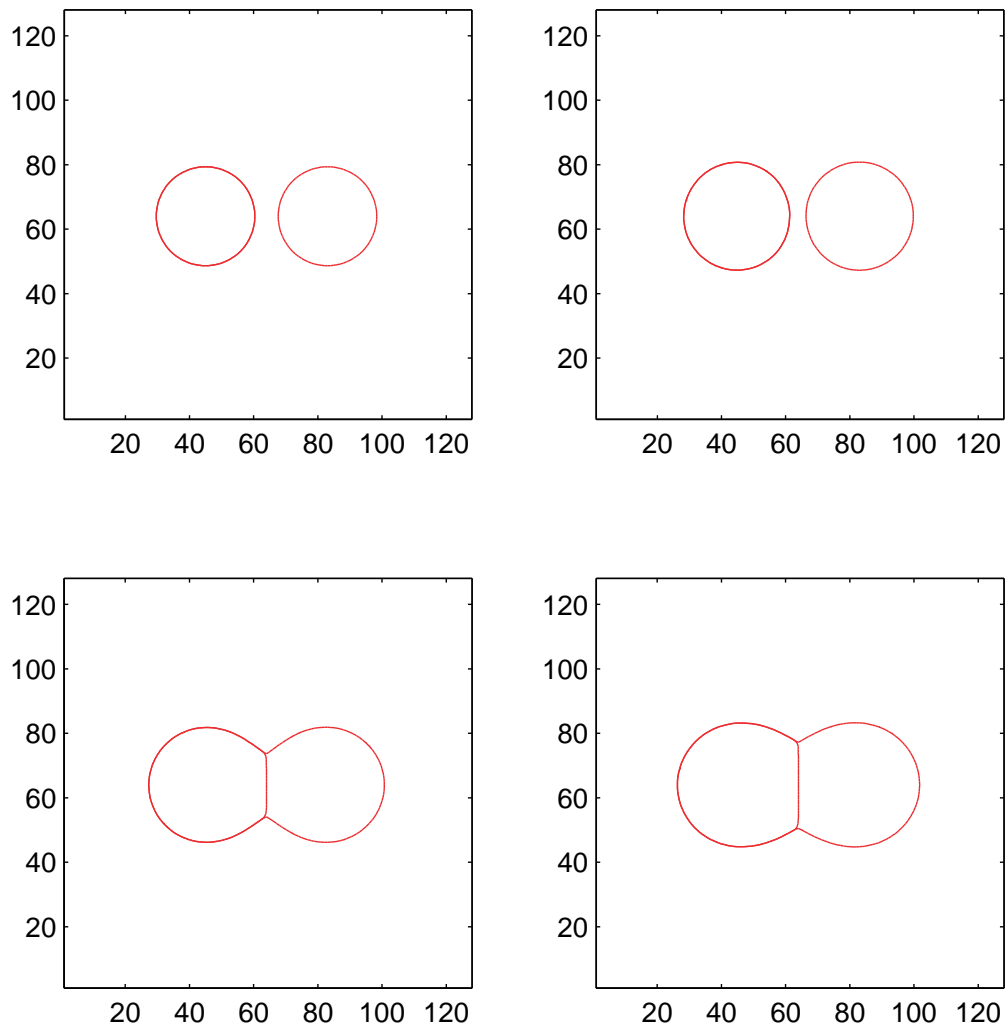

FIG. 3.8. Evolution under bulk and surface energies. Surface tensions are the same for all interfaces. The two disks represent two phases for which the bulk energy constant is $e_{1}=e_{2}=0$. Their complement, the background, which represents the third phase, has a bulk energy constant of $e_{3}=1$. The computation was carried out using the formulation in Section 3.4.1.

Next, we determine the values of $\lambda_{n}$ so that the constraints are satisfied. To begin, we make the following computation:

$$
\begin{aligned}
\frac{\partial C_{n}}{\partial t} & =\int_{n \varepsilon-\frac{\varepsilon}{2}}^{n \varepsilon+\frac{\varepsilon}{2}} \int_{\Omega} \delta(-\phi+\beta) \partial_{t} \phi d \boldsymbol{x} d \beta \\
& =\int_{n \varepsilon-\frac{\varepsilon}{2}}^{n \varepsilon+\frac{\varepsilon}{2}} \int_{\Omega} \delta(-\phi+\beta)\left[-\kappa+\sum_{m=-\infty}^{\infty} \lambda_{m} \chi_{\left\{|\phi-m \varepsilon| \leq \frac{\varepsilon}{2}\right\}}\right]|\nabla \phi| d \boldsymbol{x} d \beta \\
& =\int_{n \varepsilon-\frac{\varepsilon}{2}}^{n \varepsilon+\frac{\varepsilon}{2}} \int_{\Omega} \delta(-\phi+\beta)\left[-\kappa+\lambda_{n} \chi_{\left\{|\phi-n \varepsilon| \leq \frac{\varepsilon}{2}\right\}}\right]|\nabla \phi| d \boldsymbol{x} d \beta,
\end{aligned}
$$

Since $\partial_{t} C_{n}=0$, we find

$$
\lambda_{n}=\frac{\int_{n \varepsilon-\frac{\varepsilon}{2}}^{n \varepsilon+\frac{\varepsilon}{2}} \int_{\Omega} \delta(-\phi+\beta) \kappa|\nabla \phi| d \boldsymbol{x} d \beta}{\int_{n \varepsilon-\frac{\varepsilon}{2}}^{n \varepsilon+\frac{\varepsilon}{2}} \int_{\Omega} \delta(-\phi+\beta)|\nabla \phi| d \boldsymbol{x} d \beta} .
$$

Substitution of (4.5) into (4.4) gives the the evolution of $\phi$ that yields constrained gradient descent of $E$ with respect to the level set inner product. 
We are now going to examine the behavior of this evolution as $\varepsilon \rightarrow 0$. We divide the numerator and denominator of (4.5) by $\varepsilon$, let $g=\varepsilon n$ and take the following limit:

$$
\lim _{\varepsilon \rightarrow 0} \lambda_{n} \equiv \tilde{\lambda}_{g}
$$

where

$$
\tilde{\lambda}_{g}=\lim _{\varepsilon \rightarrow 0} \frac{\frac{1}{\varepsilon} \int_{g-\frac{\varepsilon}{2}}^{g+\frac{\varepsilon}{2}} \int_{\Omega} \delta(-\phi+\beta) \kappa|\nabla \phi| d \boldsymbol{x} d \beta}{\frac{1}{\varepsilon} \int_{g-\frac{\varepsilon}{2}}^{g+\frac{\varepsilon}{2}} \int_{\Omega} \delta(-\phi+\beta)|\nabla \phi| d \boldsymbol{x} d \beta},
$$

We note, in the sense of distributions, that

$$
\lim _{\varepsilon \rightarrow 0} \frac{1}{\varepsilon} \int_{z-\frac{\varepsilon}{2}}^{z+\frac{\varepsilon}{2}} \delta(x-y) d y=\delta(x-z)
$$

so we can rewrite (4.6) as

$$
\begin{aligned}
\tilde{\lambda}_{g} & =\frac{\int_{\Omega} \delta(-\phi+g) \kappa|\nabla \phi| d \boldsymbol{x}}{\int_{\Omega} \delta(-\phi+g)|\nabla \phi| d \boldsymbol{x}} \\
& =\text { average curvature of the } g \text {-th level curve } \\
& \equiv\langle\kappa\rangle_{g} .
\end{aligned}
$$

Finally, we observe that

$$
\lim _{\varepsilon \rightarrow 0} \sum_{n=-\infty}^{\infty} \lambda_{n} \chi_{\left\{|\phi-n \varepsilon| \leq \frac{\varepsilon}{2}\right\}}
$$

converges to a function of $\phi$, denoted $\tilde{\lambda}(\phi)$, which has the property that $\tilde{\lambda}(g)=\tilde{\lambda}_{g}$. Therefore it follows that

$$
\lim _{\varepsilon \rightarrow 0} \sum_{n=-\infty}^{\infty} \lambda_{n} \chi_{\left\{|\phi-n \varepsilon| \leq \frac{\varepsilon}{2}\right\}}=\langle\kappa\rangle_{\phi}
$$

Combining (4.9) and (4.4) yields our claim.

4.3. Numerical examples.

Equation (4.3) was solved numerically as follows. The $\langle\kappa\rangle_{\phi}$ term was evaluated by computing (4.2) for 50 values of $\beta$ and then interpolating to find $\langle\kappa\rangle_{\phi}$. The integrals in (4.2) were computed to first order using the method presented in [17]. The convection term, $-\langle\kappa\rangle_{\phi}|\nabla \phi|$, was implemented using a first order Godunov scheme and the curvature term was implemented using center differencing. The system was evolved in time using a first order explicit method. A semi-implicit algorithm similar to that found in [18] was also implemented but it was found that it did not preserve area well.

In the first example, we consider the following initial condition,

$$
\phi(x, y)=\sqrt{3 x^{2}+y^{2}+1}-1,
$$

in the domain $\Omega=[-5,5] \times[-5,5]$ with period boundary conditions. We used 300 grid points in each direction. The time step was $h^{2} / 10$ where $h$ is the mesh size. The computational results are shown in Figure 4.1. The long time behavior of (4.3) 


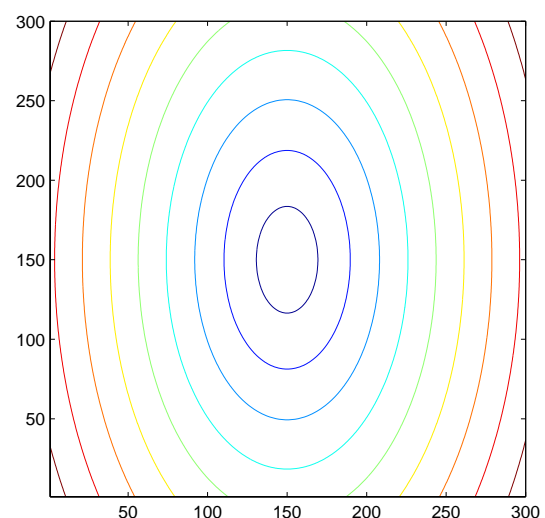

(a) initial condition

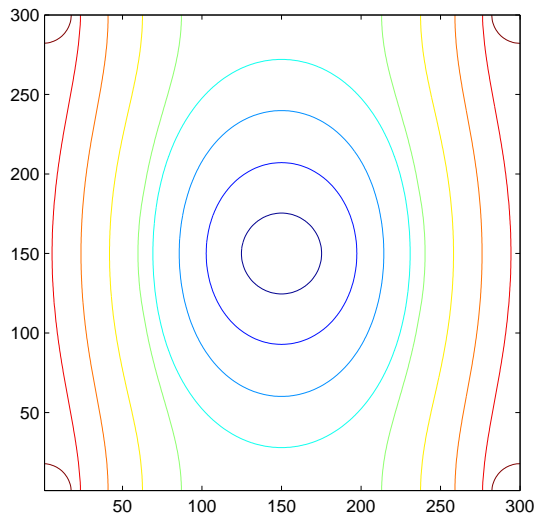

(c) time $=1.111$

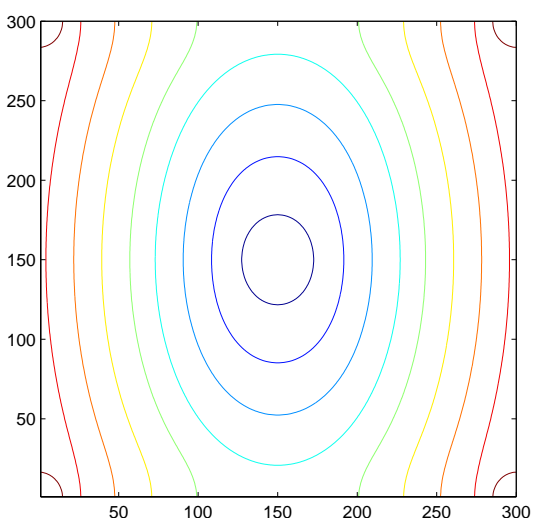

(b) time $=0.222$

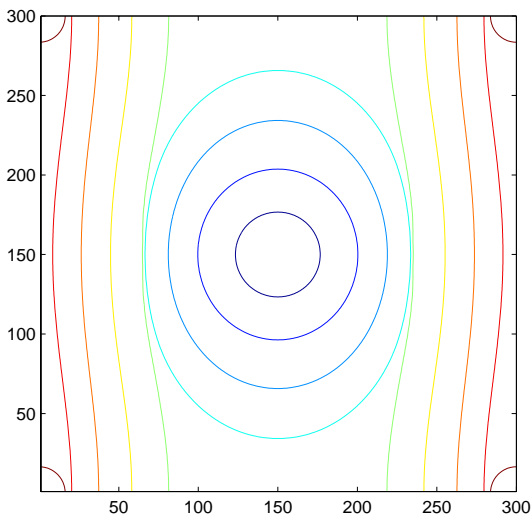

(d) time $=2.222$

FIG. 4.1. Evolution by area preserving curvature flow. In each figure, the level curves plotted are $\phi=\frac{1}{2}+n$, where $n=0, \ldots, 8$.

should result in a level set function $\phi$ whose level curves are either circles or straight lines. This seems to be the case as can be seen in Figure 4.1. However, it also seems clear that under the evolution of (4.3) various level curves can intersect. This is evident in the figure. If the level set function were allowed to become multiple valued, this interaction would result in a multiple valued level set function. However in our implementation the level set function must remain single valued, and so as a consequence we are presumably computing some sort of weak solution, not unlike a shock. A closer examination of the numerical computations shows that the intersecting level curves of these weak solutions can loose area. 


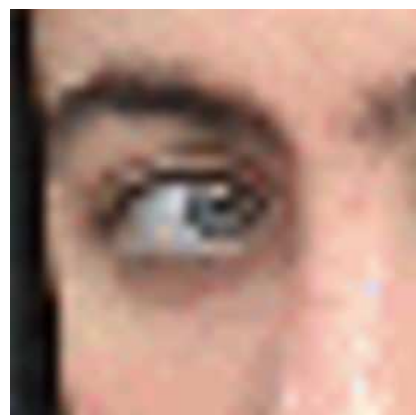

(a) $256 \times 256$ Pixelated Image

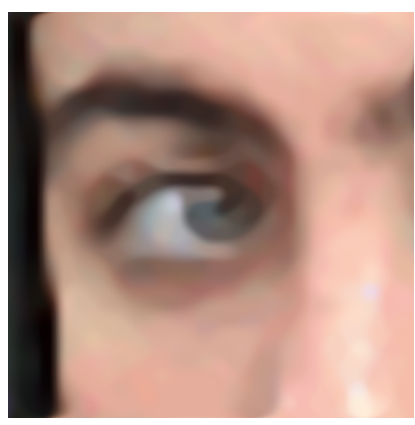

(b) After evolution by (4.3)

FIG. 4.2. Super resolution using area preserving curvature flow

In the second example, the flow given by (4.3) is used for the purpose of superresolution of an image. This is done as follows: We first start with a $64 \times 64$ image and use bilinear interpolation to create an image that is $256 \times 256$. The resulting image is shown in Figure 4.2 on the left. The underlying pixelation from the $64 \times 64$ image is evident. Next we apply (4.3) for a short time to the pixelated image in Figure 4.2. The result is shown on the right in Figure 4.2. The flow removes the pixelation without removing the small scale features; notice, for example, the eye pupil. For another approach to PDE based super-resolution, the reader is referred to [2].

\section{Summary}

In this paper we have provided a new interpretation of a well known variational formulation of curvature flow in terms of a level set description. This description shows that the level set evolution equation for curvature flow is gradient descent of the total variation norm with respect to the level set inner product. Basically, the level set inner product ensures that, when calculating Frechet derivative, variations to the level curves are measured in a geometrically meaningful way.

We also present a new level set representation of $n$ phases using $(n-1)$ phase functions. This representation has the advantage that a no overlapping/no vacuum constraint is not needed in computing geometric motions of multiple phases. This level set representation is then combined with a modified version of the variational formulation of curvature flow to obtain a gradient descent version of of a multiphase flow problem with bulk and surface energies.

In addition, we consider a level set evolution equation where each level curve has normal velocity equal to its curvature minus its average curvature: this is the area preserving curvature flow. We show that such a flow is gradient descent of the total variation norm with respect to the level set inner product, subject to an infinite number of constraints. This evolution is then used for the image processing application of super-resolution. 
Finally, we remark that all the implementations in the paper are formally first order in space and time. It would be interesting to verify this numerically and to develop higher order versions.

Acknowledgments. S. Esedoḡlu was supported in part by the National Science Foundation through Grant No. DMS-0605714. P. Smereka was supported in part by the National Science Foundation through Grant No. DMS-0509124. P. Smereka also acknowledges the support of IPAM where some of this work was completed.

\section{REFERENCES}

[1] F. Almgren, J.E. Taylor and L. Wang, Curvature-driven flows: a variational approach, SIAM J. Control and Optimization, 31(2), 387-437, 1993.

[2] M. Bertalmio, A.L. Bertozzi and G. Sapiro, Navier-Stokes, fluid dynamics, and image and video inpainting, Proceedings of the International Conference on Computer Vision and Pattern Recognition, IEEE, Kauai, HI, I, I355-I362, Dec., 2001.

[3] T.F. Chan and L.A. Vese, Active contours without edges, IEEE Transactions on Image Processing, 10, 266-277, 2001.

[4] D.G. Caraballo, A Variational Scheme for the Evolution of Polycrystals by Curvature, Ph.D. Thesis, Department of Mathematics, Princeton University, 1997.

[5] K. Deckelnick and G. Dziuk, Convergence of a finite element method for nonparametric mean curvature flow, Numer. Math., 72, 197-222, 1995.

[6] M. Droske and M. Rumpf, A level set formulation for Willmore flow, Interfaces and Free Boundaries, 6, 361-378, 2004

[7] S. Esedoḡu and R. March, Segmentation with depth but without detecting junctions, J. Math. Imaging and Vision, 18, 7-15, 2003.

[8] L. Evans and J. Spruck, Motion of level sets by mean curvature, J. Diff. Geom., 33, 635-681, 1991.

[9] H. Garcke, B. Nestler and B. Stoth, A multiphase field concept: numerical simulations of moving phase boundaries and multiple junctions, SIAM J. Appl. Math., 60, 295-315, 1999.

[10] B. Merriman, J. Bence and S. Osher, Motion of multiple junctions: a level set approach, J. Comput. Phys., 112, 334-363, 1994.

[11] M. Nitzberg and D. Mumford, The 2.1-D Sketch, Proceedings of the Third International Conference on Computer Vision, Osaka, 1990.

[12] M. Nitzberg, D. Mumford and T. Shiota, Filtering, segmentation, and depth, Lecture Notes in Computer Science, Springer-Verlag, Berlin, 662, 1993.

[13] S. Osher and R. Fedkiw, Level Set Methods and Dynamic Implicit Surfaces, Springer, New York, 2000.

[14] D. Peng, B. Merriman, S.J. Osher, H.K. Zhao and M.J. Kang, A PDE-based fast local level set method, J. Comput. Phys., 155, 410-438, 1999.

[15] F. Reitich and H.M. Soner, Three-phase boundary motion under constant velocities, I: the vanishing surface tension limit, Proc. Roy. Soc. Edin., 126A, 837-865, 1996.

[16] G. Russo and P. Smereka, A remark on computing distance functions, J. Comput. Phys., 163, $51-67,2000$.

[17] P. Smereka, A numerical approximation of a delta function with application to level set methods, J. Comput. Phys., 211, 77-90, 2006.

[18] P. Smereka, Semi-implicit level set methods for curvature flow and motion by surface diffusion, J. Sci. Comput., 19, 439-456, 2003.

[19] K.A. Smith, F.J. Solis and D.L. Chopp, A projection method for motion of triple junctions by level sets, Interfaces and Free Boundaries, 4, 263-276, 2002.

[20] J.E. Taylor, The motion of multiple junctions under prescribed phase-boundary velocities, J. Diff. Eqns., 119, 109-136, 1995.

[21] J.E. Taylor, A variational approach to crystalline triple-junction motion, J. Stat. Phys., 95, 1221-1244, 1999.

[22] L.A. Vese and T.F. Chan, A multiphase level set framework for image segmentation using the Mumford and Shah model, Int. J. Computer Vision, 50, 271-293, 2002.

[23] H.K. Zhao, S. Osher, T. Chan and B. Merriman, Variational formulation for motion of multiple junctions by level approach, UCLA CAM Report, 95-36, 1995.

[24] H.K. Zhao, T.F. Chan, B. Merriman and S. Osher, A variational level set approach to multiphase motion, J. Comput. Phys., 127, 179-195, 1996. 
[25] H.K. Zhao, B. Merriman, S. Osher and L. Wang, Capturing the behavior of bubbles and drops using the variational level set approach, J. Comput. Phys., 143, 495-518, 1998.

[26] W. Zhu, T.F. Chan and S. Esedoglu, Segmentation with depth: A level set approach, SIAM J. Sci. Comput., 28, 1957-1973, 2006. 